\title{
Accuracy of Computerized Tomography in Determining Hepatic Tumor Size in Patients Receiving Liver Transplantation or Resection
}

\author{
Raj Mittal, Charles Kowal, Thomas Starzl, David Van Thiel, Klaus Bron, Shunzaburo \\ Iwatsuki, Robert Schade, William Straub, and Andrew Dekker \\ Departments of Medicine, Pharmacology, Surgery, Radiology, and Pathology, School of \\ Medicine, University of Pittsburgh, Pittsburgh, $\mathrm{Pa}$
}

\begin{abstract}
Computerized tomography $(\mathrm{CT})$ of liver is used in oncologic practice for staging tumors, evaluating response to treatment, and screening patients for hepatic resection. Because of the impact of CT liver scan on major treatment decisions, it is important to assess its accuracy. Patients undergoing liver transplantation or resection provide a unique opportunity to test the accuracy of hepatic-imaging techniques by comparison of findings of preoperative CT scan with those at gross pathologic examination of resected specimens. Forty-one patients who had partial hepatic resection (34 patients) or liver transplantation (eight patients) for malignant (30 patients) or benign (11 patients) tumors were evaluable. Eight (47\%) of 17 patients with primary malignant liver tumors, four $(31 \%)$ of 13 patients with metastatic liver tumors, and two (20\%) of 10 patients with benign liver tumors had tumor nodules in resected specimens that were not apparent on preoperative CT studies. These nodules varied in size from 0.1 to $1.6 \mathrm{~cm}$. While 11 of 14 of these nodules were $<1.0 \mathrm{~cm}$, three of 14 were $>1.0 \mathrm{~cm}$. These results suggest that conventional CT alone may be insufficient to accurately determine the presence or absence of liver metastases, extent of liver involvement, or response of hepatic metastases to treatment.
\end{abstract}

The assessment of tumor response to treatment is an essential part of clinical oncology, as well as a critical factor in oncologic research. ${ }^{1-3}$ This function may be easily performed if the tumor has an associated serologic marker that correlates well with the extent of disease, such as in testicular cancer, gestational trophoblastic cancer, or myeloma. More frequently, however, as in liver tumors, assessment of response to treatment is dependent on the physical measurement of the tumor mass or on the use of various radiologic techniques.

Substantial variations have been reported in the physical measurement of tumor masses or liver size. ${ }^{1}$ Moreover, the usefulness of hepatomegaly in the assessment of tumor response is further compromised by recent work showing that intrahepatic metastases may enlarge without significantly changing the size of the liver. ${ }^{4}$ Various noninvasive radiologic methods have been used to assess intrahepatic lesions in an attempt to overcome the limitations of physical examination. These methods include radionuclide scintigraphy, ultrasound (US), and computerized tomography (CT). ${ }^{5}$ Radionuclide (RN) scan is more sensitive in the detection of diffuse nonneoplastic liver disease such as cirrhosis, hepatitis, and cholangitis than $\mathrm{CT}$ scans. ${ }^{6} \mathrm{CT}$, however, is superior to $\mathrm{RN}$ in the detection of biliary obstruction. ${ }^{6}$ In a comparative study of US and CT in the evaluation of focal liver disease,

(C) 1984 by American Society of Clinical Oncology.

Address reprint requests to Raj R. Mittal, MD, University of Pittsburgh, School of Medicine, 922 Scaife Hall, Pittsburgh, PA 15261.

Presented at the 19th Annual Meeting of the American Society of Clinical Oncology, held in San Diego, California, in Mal, 1983. 
US was found to be more sensitive but CT had higher specificity and provided more information about intrahepatic masses and extrahepatic disease, if present. ${ }^{7}$ Overall, when compared to either US or RN scans, CT is presently considered to be the single best examination to determine both the presence and the extent of space-occupying lesions within the liver. ${ }^{8-10} \mathrm{CT}$ scanning, therefore, has become an accepted method for defining tumor response to treatment.

Determination of the accuracy of imaging techniques in the abdomen such as CT, however, presents considerable difficulty, especially in the liver. Calibration of imaging techniques, like CT, for accuracy has depended on post-mortem examination, ${ }^{11,12}$ or on scanning artificial tumor masses. ${ }^{13}$ In patients with neoplastic disease in earlier stages, the determining of CT accuracy has not been possible.

Recent efforts at the University of Pittsburgh have focused on the use of liver transplantation, and the use of partial liver resection, such as trisegmentectomy, in the management of primary and secondary hepatic malignancies. ${ }^{14-16}$ Every patient has an abdominal CT scan performed shortly before the operative procedure. Each patient in this population, therefore, provides a unique opportunity for the study of hepatic CT-scan accuracy, since their liver is removed, and is available for subsequent study. Thus, a comparison of the tumors noted in the preoperative CT liver scan with that of the tumors noted at the time of gross pathologic examination of the resected specimen affords a measure of the accuracy of CT-liver scans.

\section{Materials and Methods}

Forty-two patients receiving liver transplantation, or partial hepatic resection, for benign or malignant tumors were studied. All patients had CT studies of the liver, with and without contrast, prior to surgery, using Conray contrast material 60\% (150 mL) given intravenously (IV). Approximately half of the contrast was administered as a rapid IV bolus and the other half was infused over five to 10 minutes. Due to a history of previous allergic reactions, one patient with hepatic hemangiomas did not receive contrast.

Imaging studies were performed on a CT scanner (model no. 8800, General Electric) with a scan time of 6.4 seconds. Serial transverse section images were obtained at 10-mm intervals, except in one patient where 15 -mm intervals were used. Patients with CT scans showing metallic-clip artifacts were considered inadequate for interpretation. One patient with a false-negative CT scan, secondary to metallic clip artifact, was excluded from the study. All patients underwent one or more additional imaging procedures. such as US. RN scan, and/or angiography at either the University of Pittsburgh or at the referring hospital.

The results of each patient's CT studies were compared with the gross findings of their resected liver specimens. Tumor nodules that were apparent on the gross pathology specimen, but were completely missed on the CT scan were noted for size, frequency, and tumor type. In patients with cholangiocarcinomas only the hepatic parenchymal nodules were evaluated for CT-scan accuracy.

\section{Results}

\section{Tumor Types}

Seventeen patients had primary hepatic malignancies: 14 hepatomas and three cholangiocarcinomas. Thirteen patients had metastatic liver tumors; 10 with colorectal carcinoma, one with hypernephroma, and two with squamous-cell carcinoma of unknown 
primary. Eleven patients had benign liver tumors; seven with hemangioma and four with hepatic adenoma.

\section{Surgical Procedures}

Patients received either a partial hepatic resection or liver transplantation. Partial hepatic resections were either trisegmentectomy or lobectomy. Forty-two surgical procedures were performed on the 41 patients in the study. Thirty-four patients underwent partial hepatic resections; 12 had lobectomy and 22 had trisegmentectomy. Eight patients received liver transplantation. One patient with multiple hepatic adenomas received two surgical procedures: a right trisegmentectomy and a liver transplantation (one-year later) for recurrent disease in the remaining left lobe.

\section{CT Scan Accuracy}

In this selected population, preoperative CT scan accurately detected the presence of nodules in the liver in all patients. CT scan detection of the extent or number of tumor nodules were less accurate than the detection of tumor presence or absence. In 27 (66\%) of 41 patients, tumor nodules were correctly detected by the CT scan. However, in 14 (34\%) of 41 patients preoperative CT did not detect all the tumor nodules and additional tumor nodules were discovered on the gross pathologic examination of the resected liver specimens (Table 1). Malignant liver tumors appeared to have a higher incidence of CT-undetected tumor nodules (12 [40\%] of 30) than did benign liver tumors (two [20\%] of 10) but the difference was statistically not significant. Eight (47\%) of 17 patients with primary malignant tumors and four $(31 \%)$ of 13 patients with liver metastases had CT-undetected tumor nodules. Figures 1 and 2 show a tumor nodule that can be seen on the resected specimen, but which was not seen on the CT-liver scan.

Table 2 shows the size of the CT-undetected tumor nodules with respect to tumor histology. These nodules were generally small, and varied in size from 0.1 to $1.6 \mathrm{~cm}$. While 11 of 14 nodules were $<1.0 \mathrm{~cm}$, three of 14 were $>1.0 \mathrm{~cm}$ in diameter.

\section{Discussion}

Scintigraphy, sonography, and CT are the various radiologic modalities used in the noninvasive evaluation of hepatic neoplasms. ${ }^{5}$ In the detection of neoplasia, CT scan generally has been reported to have a low rate of false positives (4\%-12\%) and false negatives (4\%). ${ }^{6,8} \mathrm{CT}$ scan, in contrast to $\mathrm{RN}$ scan, is more specific in differentiating between various focal hepatic lesions such as abscess/hematoma, cyst, hemangioma, and other tumors. ${ }^{17}$ Comparisons of the efficacy of these imaging modalities depends to a large extent on the current technical aspects of the modality. For example, earlier studies using CT scanners with scan times of 2.43 and 2.7 minutes respectively, claimed US to be the most accurate modality for the evaluation of hepatic space-occupying lesions. ${ }^{18,19}$ More recent studies, with faster total body scan times of 18 seconds and less roentgenogram diffraction by using narrower window widths, have shown CT to be equal, or superior, to US and RN scans in evaluating hepatic neoplasms. ${ }^{7-10}$

Cancer patients receiving hepatic resection or transplantation are an ideal population in which to study the accuracy of hepatic-imaging techniques. The imaging procedure in this setting is usually done shortly before the operative procedure. Following resection, a comparison of the resected gross pathology specimen with the preoperative imaging technique can be made. The use of a short interval between the imaging procedure and the surgical resection minimizes possible time delay artifacts in assessing the accuracy of the imaging technique. The comparison of the resected gross pathology specimen with the 
preoperative imaging technique also allows for a more detailed correlation than is possible with other techniques.

This study was undertaken to assess the accuracy of CT scans in patients with hepatic neoplasms. Our data show that a substantial proportion of the patients (34\%) had additional tumor nodules that were not detected by the preoperative CT scan and that $22 \%$ of the missed lesions were $>1.0 \mathrm{~cm}$ in diameter. Since the results of a CT scan are frequently used for making management decisions in oncologic patients, the results from our study have two implications. One involves the use of CT scans to initiate a particular therapeutic procedure, and the other involves the use of CT to evaluate tumor response secondary to treatment.

\section{CT to Initiate Therapy}

CT scan of the liver is frequently used as a staging procedure for many tumors. ${ }^{20}$ The results of this staging procedure predicates, to a large extent, the direction of subsequent treatment. For example, the presence or absence of liver metastases is of major concern in patients with primary gastrointestinal tumors. A false-negative CT scan of the liver may lead to the use of unnecessary surgical procedures or may preclude the use of specialized procedures such as hepatic artery infusion chemotherapy.

Additionally, CT liver scans may be used to stage patients as potential candidates for hepatic resection or transplant. The role of liver transplantation in the management of patients with unresectable primary hepatic tumors is still being investigated. ${ }^{15,21-27}$ Partial hepatic resection for liver metastases in certain tumors may provide for a high actuarial probability of long disease-free survival. ${ }^{21,28}$ Data from this study would suggest that $34 \%$ of the patients viewed as candidates for surgical resection by means of conventional CT may have additional tumors that may contraindicate the use of surgical resection. In this setting, intraoperative palpation of the liver, as a means of further detecting liver neoplasms may, like CT scans, also be subject to varying degrees of accuracy. Our data would indicate that about $26 \%$ of the patients will be expected to have CT-undetectable lesions that are $<1.0 \mathrm{~cm}$ in diameter. It is unclear as to the reliability or the validity of intraoperative palpation in detecting lesions $<1.0 \mathrm{~cm}$ in diameter within a hepatic parenchyma itself many centimeters in thickness. The use of $\mathrm{CT}$ alone, therefore, to select patients as candidates for surgical resection of hepatic neoplasms would seem to be insufficient and may need to be supplemented with other imaging procedures.

Hepatic angiography has been traditionally used to detect the extent and resectability of the hepatic tumors and to delineate the vascular anatomy in patients undergoing hepatic resection. ${ }^{29,30}$ Infusion hepatic angiography can enhance the parenchymal phase of the angiogram ${ }^{29,31}$ and has been reported to detect smaller hepatocellular carcinomas as compared to conventional hepatic angiography. ${ }^{32}$ Recently, CT arteriography has been shown to detect additional tumor nodules that were missed by other imaging procedures and/ or selective angiography in up to 55\% of patients, rendering initial neoplasms unresectable. ${ }^{33,34}$ Dynamic sequential CT with table incrementation during arterial portography (DSCTI-AP) has been claimed as the most sensitive means of detecting small

intrahepatic lesions, as compared to conventional imaging procedures and angiography. ${ }^{35-37}$ The lesions detected only by DSCTI-AP varied from 2 to $0.5 \mathrm{~cm}$ in diameter. ${ }^{35}$ Dynamic sequential CT with table incrementation during arterial portography is particularly useful in evaluating small tumors in the left lateral segment of the liver and invasion of the liver hilum and the portal vein. ${ }^{36,37}$

The sensitivity of CT can also be increased by hepatic specific contrast agents, such as ethiodized oil emulsion $13 .{ }^{38-42}$ Ethiodized oil emulsion 13 increases the density of normal liver and spleen parenchyma without affecting the density of the tumor. This increased 
density difference leads to detection of increased number of lesions in postcontrast scans. ${ }^{42}$ Ethiodized oil emulsion 13 is also of value in areas of the liver that usually are poorly visualized, that is, dome of the right lobe and the left lobe. ${ }^{41}$

\section{CT to Evaluate Therapy}

In contrast to the use of $\mathrm{CT}$ in the staging of patients, where the results will be used to initiate a therapeutic direction, CT may be used to evaluate the results of a current therapy. Unfortunately, little information exists as to the use of CT accuracy in determining the extent of response, either complete or partial, ${ }^{2,20}$ in the liver to treatment. Response to therapy as evaluated by CT may be determined by inspection and/or measurement of a particular tomographic section or by calculation of the entire tumor volume across all tomographic sections in which it appears. It has been reported that evaluation of individual tomographic sections without calculating the entire volume is frequently inaccurate and can miss a $20 \%-80 \%$ (range, $20-150 \mathrm{~mL}$ ) change in initial absolute volume. ${ }^{28}$ In contrast, CTtumor volume calculations are more accurate,${ }^{4,8,43}$ but are also more time consuming and are not always readily available to the clinician. Determination of the accuracy of CT in evaluating tumor responses in the liver is important since the reporting of high response rates (up to 83\%) is currently being used as part of the rationale for the treatment of patients with HA1 chemotherapy. ${ }^{21,44,45}$ Data from our study would further suggest that CT alone is insufficient to establish that patients with previously identifiable disease have achieved a complete response, or complete disappearance of measurable disease, in response to treatment.

In conclusion, CT of the liver with and without IV contrast can miss tumor nodules up to 1.6 $\mathrm{cm}$ in $34 \%$ of the patients studied. Until technical capabilities improve to detect lesions <2 $\mathrm{cm}$ reliably, conventional $\mathrm{CT}$ findings alone may be insufficient to accurately determine the presence or absence of liver metastases, extent of liver involvement, or response of hepatic metastases to treatment.

\section{References}

1. Moertel CG, Hanley JA. The effect of measuring error on the results of therapeutic trials in advanced cancer. Cancer. 1976; 38:388-394. [PubMed: 947531]

2. Kaplan, BH. Measuring the Effect of Anti-Cancer Treatment. Wilmington: Stuart Pharmaceuticals; 1981.

3. Lavin PT, Flowerdew G. Studies in variation associated with the measurement of solid tumors. Cancer. 1980; 46:1286-1290. [PubMed: 7214310]

4. Ettinger DS, Leichner PK, Siegelman S, et al. Computed tomography (CT) associated volumetric analysis of primary liver tumors as a measure of response to therapy. Proc Am Soc Clin Oncol. 1983; 2:115. Abstr.

5. Bernadino ME, Lewis E. Imaging hepatic neoplasms. Cancer. 1982; 50:2666-2671. [PubMed: 7139560]

6. MacCarty RL, Wahner HW, Stephens DH, et al. Retrospective comparison of radionuclide scans and computed tomography of the liver and pancreas. AJR. 1977; 129:23-28. [PubMed: 409138]

7. Bartolozzi C, Ciatti S, Lucarelli E, et al. Ultrasound and computer tomography in the evaluation of focal liver disease. Acta Radiol [Diagn]. 1981; 22:545-548.

8. Snow JH, Goldstein HM, Wallace S. Comparison of scintigraphy sonography and computerized tomography in the evaluation of hepatic neoplasms. AJR. 1979; 132:915-918. [PubMed: 108968]

9. Knopf DR, Torres WE, Fajman WJ, et al. Comparative accuracy of scintigraphy and computer tomography. AJR. 1982; 138:623-627. [PubMed: 6978022]

10. Alderson PO, Adams DF, McNeil BJ, et al. Computed tomography, ultrasound, and scintigraphy of the liver in patients with colon or breast carcinoma: A prospective comparison. Radiology. 1983; 149:225-230. [PubMed: 6611929] 
11. Husband, J.; Hobday, P.; Cassell, KJ. Quantitation of therapeutic response. In: Husband, JE.; Hobday, PA., editors. Computerized Axial Tomography in Oncology European Seminar on Computerized Axial Tomography in Clinical Practice. New York: Churchill Livingstone; 1981.

12. Lamarque, JL.; Bruel, JM.; Dondelinger, R., et al. Liver and pancreas. In: Baert, A.; Jenmart, L.; Wackenheim, A., editors. Clinical Computer Tomography; Head and Trunk Meeting of College d'enseignment post-universitare de radiologie; Luxembourg. 1977; New York: Springer Verlag; 1978.

13. Husband JE, Casell KJ, Packham MJ, et al. The role of computed tomography in the assessment of tumour volume in patients with malignant testicular teratoma. Br J Radiol. 1981; 54(Suppl 15):5053. [PubMed: 7448501]

14. Starzl TE, Iwatsuki SE, Shaw BW Jr, et al. Left hepatic trisegmentectomy. Surg Gynecol Obstet. 1982; 155:21-27. [PubMed: 6283687]

15. Iwatsuki S, Klintmalm GB, Starzl TE. Total hepatectomy and liver replacement (orthotopic liver transplantation) for primary hepatic malignancy. World J Surg. 1982; 6:81-85. [PubMed: 7046266]

16. Iwatsuki S, Shaw BW, Starzl TE. Experience with 150 liver resections. Ann Surg. 1983; 197:247253. [PubMed: 6830332]

17. Biello DR, Levitt RG, Siegel BA, et al. Computed tomography and radionuclide imaging of the liver: A comparative evaluation. Radiology. 1978; 127:159-163. [PubMed: 635176]

18. Grossman Z, Wistow BW, Bryan P, et al. Radionuclide imaging, computed tomography and gray scale ultrasonography of the liver: A comparative study. J Nucl Med. 1977; 18:327-332.

[PubMed: 191578]

19. Bryan PJ, Dinn MW, Grossman ZD, et al. Correlation of computed tomography, Gray scale ultrasonography, and radionuclide imaging of the liver in detecting space-occupying processes. Radiology. 1977; 124:387-393. [PubMed: 195306]

20. Smalley RV, Malmud LS, Ritchie WGM. Pre-operative scanning: Evaluation for metastatic disease in carcinoma of the breast, lung, colon, bladder and prostate. Semin Oncol. 1980; 7:358-369. [PubMed: 7008200]

21. Niederhuber JE, Ensminger WD. Surgical considerations in the management of hepatic neoplasia. Semin Oncol. 1983; 10:135-147. [PubMed: 6306833]

22. Starzl TE, Koep LJ, Halgrimson CJ, et al. Fifteen years of clinical liver transplantation. Gastroenterology. 1979; 77:375-388. [PubMed: 376395]

23. Starzl TE, Koep LJ. Surgical approaches for primary and metastatic liver neoplasms including total hepatectomy with orthotopic liver transplantation. Prog Clin Cancer. 1978; 7:181-193. [PubMed: 360276]

24. Starzl TE, Koep L, Porter KA, et al. Decline in survival after liver transplantation. Arch Surg. 1980; 115:815-819. [PubMed: 6992740]

25. Calne RY, Williams R. Liver transplantation. Curr Probl Surg. 1979; 16:3-44.

26. Calne RY. Transplatation of the liver. Ann Surg. 1978; 188:129-138. [PubMed: 99096]

27. Starzl TE, Klintmalm GB, Femandez-Bueno C. Past and future prospects of orthoptic liver transplantation. Arch Surg. 1981; 116:1342-1343. [PubMed: 7025801]

28. Foster JH. Survival after liver resection for secondary tumors. Am J Surg. 1978; 135:389-394. [PubMed: 626320]

29. Freeny PC. Angiography of hepatic neoplasma. Semin Roentgenol. 1983; 18:114-122. [PubMed: 6306837]

30. Gui L, Xin-Ye H, Jin G, et al. Selective angiography in diagnosing primary hepatic carcinoma. Chin Med J. 1982; 95:667-673. [PubMed: 6295715]

31. Rosch J, Freeny PC, Antonovic R, et al. Infusion hepatic angiography in diagnosis of liver metastases. Cancer. 1976; 38:2278-2286. [PubMed: 1000468]

32. Takashima T, Matsuui O. Infusion hepatic angiography in the detection of small hepatocellular carcinomas. Radiology. 1980; 136:321-325. [PubMed: 6250195]

33. Freeny PC, Marks WM. Computed tomographic arteriography of the liver. Radiology. 1983; 148:193-197. [PubMed: 6304808] 
34. Prando A, Wallace S, Bernadino ME, et al. Computed tomographic arteriography of the liver. Radiology. 1979; 130:697-701. [PubMed: 218242]

35. Matsui O, Kadoya M, Suzuki M, et al. Work in Progress: Dynamic sequential computed tomography during arterial portography in the detection of hepatic neoplasms. Radiology. 1983; 146:721-727. [PubMed: 6298857]

36. Nakao N, Miura K, Takayasu Y, et al. CT angiography in hepatocellular carcinoma. J Comput Assist Tomogr. 1983; 7:780-787. [PubMed: 6309927]

37. Clark RA, Matsui O. CT of liver tumors. Semin Roentgenol. 1983; 18:149-162. [PubMed: 6306840]

38. Vermess M, Doppman JL, Sugarbaker P, et al. Clinical trials with a new intravenous liposoluble contrast material for computed tomography of the liver and spleen. Radiology. 1980; 137:217222. [PubMed: 6252571]

39. Vermess M, Doppman JL. CT of the liver with intravenous lipid contrast material: Review of the current status. Semin Roentgenol. 1983; 18:102-105. [PubMed: 6306836]

40. Miller DL, Rosenbaum RC, Sugarbaker PH, et al. Detection of hepatic metastases: Comparison of EOE-13 computed tomography and scintigraphy. AJR. 1983; 141:931-935. [PubMed: 6312788]

41. Vermess M, Doppman JL, Sugarbaker PH, et al. Computed tomography of the liver and spleen with intravenous lipoid contrast material: Review of 60 examinations. AJR. 1982; 138:1063-1071. [PubMed: 6282100]

42. Lewis E, Aufdersheide JF, Bernardino ME, et al. CT detection of hepatic metastases with ethiodized oil emulsion 13. J Comput Assist Tomogr. 1982; 6:1108-1114. [PubMed: 6294154]

43. Brenner DE, Whitley N, Houk T, et al. Tumor volume measurement by computed tomography. Proc Am Soc Clin Oncol. 1981:22-347. Abstr.

44. Ansfield FJ, Ramirez G, Davis HL, et al. Further cancer studies with intrahepatic arterial infusion with 5-fluorouracil. Cancer. 1975; 36:2413-2417. [PubMed: 1212659]

45. Reed ML, Vaitkevicius VK, Al-Sarraf M, et al. The practicality of chronic hepatic artery infusion therapy of primary and metastatic hepatic malignancies: Ten-year results of 124 patients in a prospective protocol. Cancer. 1981; 47:402-409. [PubMed: 6257376] 

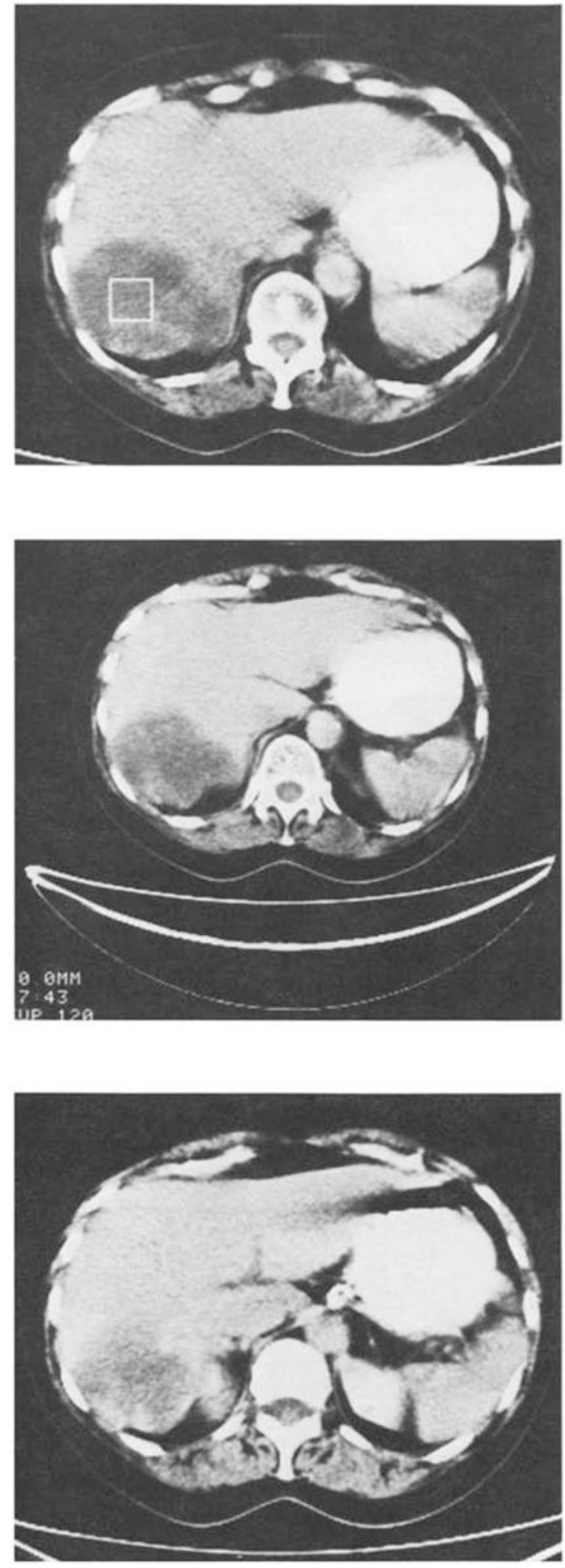

Fig. 1.

Top, middle, and bottom: CT scan of the liver showing a single tumor mass in the right lobe of the liver. 


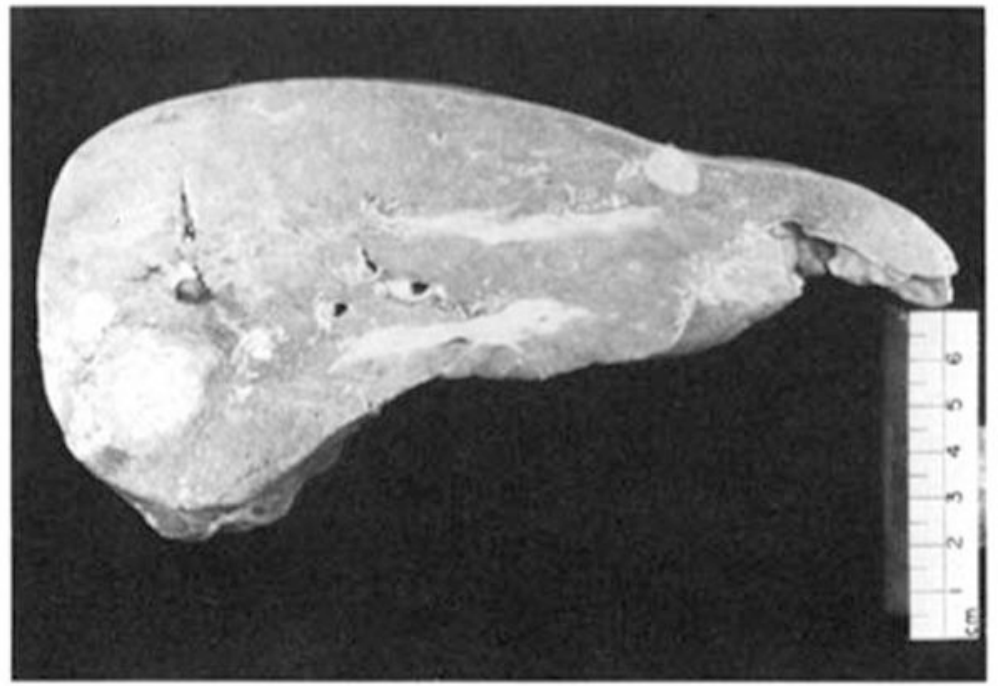

Fig. 2.

Resected liver specimen showing an additional tumor nodule near anterior surface of the liver that was not seen on the CT liver scan. 
Table 1

Patients with Additional Tumor Nodules Undetected by Preoperative CT Scan

\begin{tabular}{lll}
\hline Tumor Type & Histology & No. Patients with Undetected Nodules/Total No. of Patients \\
\hline Malignant & & $7 / 14$ \\
Primary & Hepatoma & $1 / 3$ \\
& Cholangiocarcinoma & $8 / 17(47)$ \\
& Total & $4 / 10$ \\
Metastatic & Colon & $0 / 2$ \\
& Squamous-cell carcinoma & $0 / 1$ \\
& Renal & $4 / 13(31)$ \\
& Total & $2 / 7$ \\
Benign & Hemangioma & $0 / 4$ \\
& Adenoma & $2 / 11(18)$ \\
& Total & $14 / 41(34)$ \\
\hline
\end{tabular}

NOTE. Data in parentheses are percentage. 
Table 2

\section{Correlation of Tumor Type and Undetected Nodule Size}

\begin{tabular}{|c|c|c|}
\hline \multirow[b]{2}{*}{ Tumor Type } & \multicolumn{2}{|c|}{ Nodule Size } \\
\hline & $<1 \mathrm{~cm}$ & $>1 \mathrm{~cm}$ \\
\hline Hepatoma & 6 & $1^{*}$ \\
\hline Cholangiocarcinoma & 1 & 0 \\
\hline Colon-metastatic & 2 & $2^{\dagger}$ \\
\hline Hemangioma & 2 & 0 \\
\hline Total & 11 & 3 \\
\hline \multicolumn{3}{|c|}{ NOTE. Data are no. of patients. } \\
\hline
\end{tabular}

